\title{
Effect of PCSK9 inhibitors on pulse wave velocity and monocyte-to-HDL-cholesterol ratio in familial hypercholesterolemia subjects: results from a single-lipid-unit real-life setting
}

\author{
Roberto Scicali ${ }^{1} \cdot$ Antonino Di Pino $^{1} \cdot$ Viviana Ferrara $^{1} \cdot$ Agata Maria Rabuazzo $^{1}$ - Francesco Purrello ${ }^{1}$. \\ Salvatore Piro ${ }^{1}$
}

Received: 3 February 2021 / Accepted: 3 March 2021 / Published online: 21 March 2021

(c) The Author(s) 2021

\begin{abstract}
Aims Subjects with familial hypercholesterolemia (FH) are characterized by an increased amount of low-density lipoprotein cholesterol (LDL-C) that promotes a continuous inflammatory stimulus. Our aim was to evaluate the effect of PCSK9-i on inflammatory biomarkers, neutrophil-to-lymphocyte ratio, monocyte-to-high-density lipoprotein ratio (MHR), and on early atherosclerosis damage analyzed by pulse wave velocity (PWV) in a cohort of FH subjects.

Methods In this prospective observational study, we evaluated 56 FH subjects on high-intensity statins plus ezetimibe and with an off-target LDL-C. All subjects were placed on PCSK9-i therapy and obtained biochemical analysis as well as PWV evaluation at baseline and after six months of PCSK9-i therapy.

Results After six months of add-on PCSK9-i therapy, only 42.9\% of FH subjects attained LDL-C targets. As expected, a significant reduction of LDL-C $(-49.61 \%, p<0.001)$ was observed after PCSK9-i therapy. Neutrophil count (NC) and MHR were reduced by PCSK9-i $(-13.82 \%$ and $-10.47 \%$, respectively, $p$ value for both $<0.05)$ and PWV significantly decreased after PCSK9-i therapy $(-20.4 \%, p<0.05)$. Finally, simple regression analyses showed that $\Delta$ PWV was significantly associated with $\Delta$ LDL-C $(p<0.01), \Delta$ NC and $\Delta$ MHR ( $p$ value for both $<0.05)$.

Conclusions In conclusion, PCSK9-i therapy significantly improved lipid and inflammatory profiles and PWV values in FH subjects; our results support the positive effect of PCSK9-i in clinical practice.
\end{abstract}

Keywords Familial hypercholesterolemia · PCSK9 inhibitors · Pulse wave velocity · Innate immunity · Inflammatory profile $\cdot$ Cardiovascular risk

\section{Introduction}

Atherosclerosis is a long-term damaging process and its progression leads to cardiovascular system injury [1]. Several environmental and genetic factors play a part in atherosclerosis progression; among these, an increased plasma level of low-density lipoprotein LDL cholesterol (LDL-C)

Managed by Antonio Secchi.

Francesco Purrello

francesco.purrello@unict.it

1 Department of Clinical and Experimental Medicine, University of Catania, Internal Medicine, Garibaldi Hospital, Via Palermo 636, 95122 Catania, Italy is causatively associated with atherosclerotic cardiovascular disease (ASCVD) [2]. However, the full definition of atherosclerosis as a chronic deposition of LDL-C may be not exhaustive; in fact, other processes are involved in the pathogenesis and progression of atherosclerosis together with LDL cholesterol.

In the last few years, several studies have indicated the importance of an inflammatory state in the pathophysiology of ASCVD [3, 4]; in particular, inflammation appears to be the final expression of the systemic interaction between hypercholesterolemia and the immune system during atherosclerosis progression [5]. In subjects with hypercholesterolemia, previous studies showed that lipid storage in the arterial wall promoted the inflammatory cascade and thus the migration of immune cells such as monocyte-derived 
macrophages and $\mathrm{T}$ lymphocyte subtypes into the inflammatory lipid-wall injury [6, 7]. The concept of atherosclerosis as a continuous inflammatory process promoted by a persistent LDL-C level and immune system activation may explain why, despite lifestyle changes and lipid-lowering treatments (LLT), ASCVD is still considered the leading cause of disease burden and death [8]. Increasing attention has been recently focused on novel inflammatory biomarkers largely available in clinical practice such as neutrophilto-lymphocyte ratio (NLR) and monocyte-to-high-density lipoprotein ratio (MHR); in fact, several studies showed that these inflammatory biomarkers satisfactorily predicted ASCVD in the general population $[9,10]$. In this context, NLR and MHR may be useful to better discriminate the "athero-inflammatory" profile in the general population and, in particular, in subjects with high LDL-C levels such as familial hypercholesterolemia (FH) [11].

$\mathrm{FH}$ is the most frequent monogenic genetic disorder characterized by an increased LDL-C level from childhood [12]. If not treated, FH subjects experience both an increase of pulse wave velocity (PWV) and subsequently premature ASCVD [13]; thus, early diagnosis and LLT are needed to ameliorate the cardiovascular prevention in $\mathrm{FH}$ subjects [14]. However, despite intensive statin therapy, few FH subjects obtain the recommended lipid targets, while ASCVD affects the majority of FH subjects $[15,16]$.

In the era of novel LLT, more attention has been paid to the inhibitors of proprotein convertase subtilisin/kexin type 9 (PCSK9-i) [17]. PCSK9-i leads to a reduction of circulating PCSK9 and thus reduces LDL receptor cleavage and enhances its presence on the liver cell surface [18]. The clinical efficacy of PCSK9-i was shown in previous studies $[19,20]$; in particular, the reduction of LDL-C and ASCVD by PCSK9-i was $\simeq 50-60 \%$ and $15 \%$, respectively. Because of its cardiovascular properties, PCSK9-i therapy is a crucial LLT in subjects at high cardiovascular risk such as FH [21]. No data exist regarding the effect of PCSK9-i on NLR and MHR in these subjects.

In this study, we aimed to evaluate the effect of PCSK9$i$ on the inflammatory biomarkers NLR and MHR and on early atherosclerosis damage evaluated by pulse wave velocity (PWV) in a cohort of FH subjects.

\section{Methods}

\section{Study design and population}

This was an open-label, prospective, observational study involving subjects with a previously confirmed FH genetic diagnosis [22]. All participants were enrolled from the Lipid Centre of the Garibaldi Hospital/University of Catania, Sicily, Italy, from October 2018 to September 2020.
All participants were aged between 18 and 70 years and were on high-intensity statins (atorvastatin $40-80 \mathrm{mg}$, rosuvastatin $20-40 \mathrm{mg}$ ) plus ezetimibe for at least six months at the time of enrollment. Finally, all participants had an LDL-C beyond the recommended targets according to the European Society of Cardiology/European Atherosclerosis Society guidelines 2019 for the management of dyslipidemias. After a 12-h fast, all participants had a hematological and clinical evaluation. For all participants, biochemical analyses and PWV evaluations were performed at baseline (T0) and after six months (T1) of PCSK9-i therapy. Body weight and height were measured, and body mass index (BMI) was calculated as weight divided by the squared value of height $(\mathrm{kg} / \mathrm{m} 2)$ [23]. Arterial hypertension was defined as brachial blood pressure (BP) $\geq 140 \mathrm{~mm} \mathrm{Hg}$ (systolic) and/or $90 \mathrm{~mm} \mathrm{Hg}$ (diastolic) on at least two different occasions, or if the subjects were on antihypertensive therapy [23]. Statin therapy was defined as a daily intake of statins; duration of statin therapy was defined as the numbers of years on statin therapy [24]. Non-statin lipid-lowering therapy was defined as the intake of lipid-lowering treatments other than statins (niacin, bile acid sequestrants, fibrates, nutraceuticals) [24]. Type 2 diabetes (T2D) was defined as a fasting plasma glucose $\geq 126 \mathrm{mg} / \mathrm{dL}$ on two consecutive readings or a glycated hemoglobin (HbA1c) $\geq 6.5 \%$ or the use of antidiabetic medications [25]. Smokers were divided into either current smoker (defined as any cigarette in the last month) or past smoker [26]. ASCVD was defined as documented previous myocardial infarction, acute coronary syndrome, coronary revascularization (percutaneous coronary intervention or coronary artery bypass graft surgery) or other arterial revascularization procedures, stroke or transient ischemic attack, or atherosclerotic artery disease [27]. LDL-C target was defined as an LDL-C $<70 \mathrm{mg} / \mathrm{dl}$ for FH subjects without ASCVD or an LDL-C $<55 \mathrm{mg} / \mathrm{dl}$ for FH subjects with ASCVD [28]. Exclusion criterion was the use of non-statin lipid-lowering therapy.

\section{Biochemical analysis}

Fasting plasma glucose (FPG) as well as serum total cholesterol (TC), TG, high-density lipoprotein cholesterol (HDLC), high-sensitivity C-reactive protein (hs-CRP), aspartate transaminase (AST), alanine transaminase (ALT) and creatine phosphokinase (CPK) were assessed by available enzymatic methods [29]. Apolipoprotein B (ApoB) and apolipoprotein A1 (ApoA1) were evaluated with a nephelometer assay (Siemens AG Healthcare Sector, Erlangen, Germany) [30]. Levels of lipoprotein (a) [Lp(a)] were measured with the latex agglutination immunoassay [31]. LDL-C was obtained by the Friedewald formula. Non-HDL cholesterol (non-HDL-C) was derived from baseline values. HbA1c was measured with high-performance liquid chromatography 
using a National Glycohemoglobin Standardization Program and standardized to the Diabetes Control and Complications Trial assay [32]. Chromatography was performed using a certified automated analyzer (HPLC; HLC-723G7 hemoglobin HPLC analyzer; Tosoh Corp.; normal range $4.25-5.9 \%$ [23-41 $\mathrm{mmol} / \mathrm{mol}])$. White blood cell count was performed by a blood cell analyzer (UniCel DxH-900, Beckman Coulter, Milan, Italy). NLR and MHR were derived from baseline values.

\section{Pulse wave velocity evaluation}

The SphygmoCor CVMS (AtCor Medical, Sydney, Australia) system was used for PWV evaluation. This system is made up of a tonometer and 2 different pressure waves recorded at the common carotid artery (proximal recording site) and at the femoral artery (distal recording site). The distance between the recording sites and the suprasternal notch was measured by a tape measure. An electrocardiogram was performed to determine the start of the pulse wave. The PWV was defined as the difference in the time interval of the pulse wave between the 2 different recording sites and the heart, divided by the travel distance of the pulse waveform. The PWV was calculated on the mean of 10 consecutive pressure waveforms to perform a complete respiratory cycle [33].

\section{Statistical analysis}

The distributional characteristics of each variable, including normality, were assessed by the Kolmogorov-Smirnov test. Data are reported as mean \pm standard deviation (SD) for continuous parametric parameters and median (interquartile range-IQR) for continuous nonparametric variables and as frequency (percentage) for categorical variables. When necessary, continuous nonparametric variables (TG, Lpa, CPK, duration of statin therapy and hs-CRP) were logarithmically transformed to reduce skewness. The $\chi 2$ test was used for categorical variables. The changes of TC, HDLC, TG, LDL-C, non-HDL-C, ApoB, ApoAI, ApoB/ApoAI, Lpa, FPG, HbA1c, BMI, systolic BP, diastolic BP, hs-CRP, CPK, GOT, GPT, NLR, MHR and PWV, from baseline (T0) for all the treatment time (T1 vs T0), were evaluated as delta $(\Delta)$ and calculated according to the following formula: [(T1$\left.\mathrm{T} 0) / \mathrm{T} 0)^{*} 100\right]$. To test time-dependent differences (T1 vs T0) of clinical and biochemical characteristics in the study population, we used Student's t test. Finally, simple regression analyses were performed to relate $\Delta \mathrm{PWV}$ with $\Delta \mathrm{LDL}-\mathrm{C}$, $\Delta$ NLR and $\triangle$ MHR. All statistical analyses were performed using IBM SPSS Statistics package for Windows version 23. For all tests, $\mathrm{p}<0.05$ was considered significant.

The study was approved by the local ethics committee (prot. Number 46/19) in accordance with the ethical standards of the institutional and national research committees and with the 1964 Declaration of Helsinki and its later amendments or comparable ethical standards. Informed consent was obtained from each subject enrolled in the study.

\section{Results}

In total, $245 \mathrm{FH}$ subjects were evaluated; of these, 56 subjects satisfied the inclusion criteria and participated in this open-label, prospective, observational study. According to the lipid-lowering recommendation of the European Society of Cardiology/European Atherosclerosis Society guidelines 2019 for the management of dyslipidemias and the Italian reimbursement rules of PCSK9-i, all FH subjects started PCSK9-i therapy; in particular, 4 subjects added alirocumab $75 \mathrm{mg}, 24$ subjects added alirocumab $150 \mathrm{mg}$, and 28 subjects added evolocumab $140 \mathrm{mg}$ (Fig. 1).

Table 1 shows the characteristics of the study population; $46.4 \%$ of FH subjects had a prior ASCVD. All subjects were heterozygous $\mathrm{FH}$, and the majority of subjects had an LDLR genetic variant; furthermore, more subjects were on rosuvastatin $20 \mathrm{mg}$ than atorvastatin $40 \mathrm{mg}$ and $39.3 \%$ of subjects were on antihypertensive therapy.

After six months of add-on PCSK9-i therapy, only $42.9 \%$ of FH subjects attained LDL-C targets according to the European Society of Cardiology/European Atherosclerosis Society guidelines 2019 for the management of dyslipidemias. As expected, PCSK9-i significantly reduced TC, LDL-C, non-HDL-C and ApoB plasma levels (- 35.35\%, $-49.61 \%,-44.16 \%$ and $-43.36 \%$, respectively), whereas no difference was found on TG, HDL-C, ApoAI and Lp (a). Furthermore, glucose profile was unvaried after adding-on PCSK9-i therapy; at baseline $3 \mathrm{FH}$ subjects were diabetics and no new cases of T2D were detected after adding-on PCSK9-i therapy. BMI, as well as systolic and diastolic BP, was unchanged after adding-on PCSK9-i therapy; moreover, liver and muscle enzymes were similar before and after PCSK9-i therapy (Table 2).

Table 3 shows the white blood cell count and inflammatory profiles after six months of PCSK9-i therapy. PCSK9$i$ therapy significantly reduced the neutrophil count (NC) $\left(5.21 \pm 1.04\right.$ vs $\left.4.49 \pm 0.95 \times 10^{3} / \mu \mathrm{L}[\Delta-13.82 \%], p<0.05\right)$, while the monocyte count was reduced without reaching statistical significance; furthermore, the lymphocyte count was unchanged after six months of PCSK9-i therapy. As concerns the inflammatory profile, PCSK9-i therapy significantly reduced MHR $(11.56 \pm 4.14$ vs $10.35 \pm 4.16[\Delta$ $-10.47 \%$ ], $p<0.05$ ), while hs-CRP values were similar before and after PCSK9-i therapy.

As illustrated in Fig. 2, PWV significantly ameliorated after six months of PCSK9-i therapy $(9.48 \pm 1.45$ vs $7.55 \pm 1.43 \mathrm{~m} / \mathrm{s}[\Delta-20.4 \%], p<0.01)$. 
Fig. 1 Enrollment flowchart of the study population. $\mathrm{FH}=$ familial hypercholesterolemia, PCSK9-i = proprotein convertase subtilisin/kexin type 9 inhibitors
$245 \mathrm{FH}$ patients were evaluated

76 patients aged under 18 or over 70 were excluded

$169 \mathrm{FH}$ patients were included

18 patients assuming also non statin lipid lowering therapies were excluded

$151 \mathrm{FH}$ patients were included

36 patients not on high intensity statin therapy plus ezetimibe were excluded

$115 \mathrm{FH}$ patients were included

59 patients already on LDL-C target were excluded

\section{$56 \mathrm{FH}$ patients participated in the study and started \\ PCSK9-i (4 pts alirocumab $75 \mathrm{mg} / 24$ pts alirocumab $150 \mathrm{mg} / 28 \mathrm{pts}$ evolocumab $140 \mathrm{mg}$ )}

Finally, simple regression analyses showed that $\Delta \mathrm{PWV}$ was significantly associated with $\Delta$ LDL-C $(p<0.01), \Delta \mathrm{NC}$ and $\Delta$ MHR ( $p$ value for both $<0.05)$ (Table 4).

\section{Discussion}

Over the last few years, increasing attention has been given to the synergic role of LDL-C and inflammatory status in atherosclerotic injury; the coexistence of these atherogenic conditions may be crucial in subjects at high cardiovascular risk such as FH subjects. In these subjects, LDL-C appears to be the main trigger of inflammatory status that promotes the migration of innate immune cells such as monocytes and neutrophils to the atherosclerotic lesion [34, 35]; in line with these considerations, previous studies showed that $\mathrm{FH}$ subjects were more inflamed than non-FH subjects and statin therapy was not able to reduce this difference $[13,36]$. In this context, novel lipid-lowering strategies such as PCSK9-i may be useful to reduce LDL-C and inflammatory status in FH subjects.

In our study, we analyzed the effect of PCSK9-i on lipid profile, white blood cell count, inflammatory status and pulse wave velocity in FH subjects; to the best of our knowledge, this is the first study evaluating the role of PCSK9-i on this atherogenic axis in this population. We found that
LDL-C, NC, MHR and PWV were significantly reduced after six months of adding-on PCSK9-i therapy; furthermore, simple regression analyses showed that $\Delta \mathrm{PWV}$ was significantly associated with $\Delta$ LDL-C, $\Delta$ NC and $\Delta$ MHR.

Our findings may be related to the putative role of PCSK9 as an immune mediator in the atherosclerotic process [37]; in fact, by increasing the vascular endothelial cell expression of lectin-like oxidized low-density lipoprotein receptor-1 (LOX-1), PCSK9 plasma levels activate an inflammatory cascade promoting the migration of neutrophils and monocytes in the atherosclerotic lesion [38]. In line with these findings, Li et al. previously showed that PCSK9 plasma levels were positively associated with white blood cell count and its subtypes in subjects with coronary artery disease [39]; furthermore, Ricci et al. showed that PCSK9 promoted a pro-inflammatory stage in monocyte-derived macrophages [40]. Thus, the inhibition of PCSK9 plasma levels may diminish the risk of ASCVD by reducing LDL-C and white blood cell subtypes; in line with these considerations, in our study PCSK9-i therapy significantly reduced LDL-C and $\mathrm{NC}$ in FH subjects.

As regards the inflammatory profile, previous studies showed that PCSK9-i did not reduce hs-CRP levels in the general population [41] and the same finding was observed in our FH cohort; however, Kuhnast et al. showed that these drugs could suppress the inflammatory state by reducing 
Table 1 Characteristics of the study population. Data are presented as mean \pm standard deviation, percentages, or median (interquartile range). $\mathrm{FH}=$ familial hypercholesterolemia, $\mathrm{ASCVD}=$ atherosclerotic cardiovascular disease, LDLR=low-density lipoprotein receptor, $\mathrm{ApoB}=$ apolipoprotein $\mathrm{B}$

\begin{tabular}{ll}
\hline Demographic characteristics & \\
\hline $\mathrm{N}$ & 56 \\
Age, years & $56.38 \pm 6.89$ \\
Men, $n(\%)$ & $28(50)$ \\
Smokers, $n(\%)$ & $12(21.4)$ \\
ASCVD, $n(\%)$ & $26(46.4)$ \\
FH genotype & \\
LDLR, $n(\%)$ & \\
$55(98.2)$ & $1(1.8)$ \\
ApoB, $n(\%)$ & \\
Mutation class & \\
Amino acid change, $n(\%)$ & \\
$30(53.6)$ & $26(46.4)$ \\
Null allele, $n(\%)$ & \\
FH phenotype & \\
Heterozygous FH, $n(\%)$ & \\
Treatment & \\
Duration of statin therapy, years & \\
12 (2-20) & \\
Duration of ezetimibe therapy, years & \\
Antihypertensive therapy, $n(\%)$ & \\
High-intensity statin therapy & \\
Atorvastatin 40 mg, $n(\%)$ & \\
Rosuvastatin 20 mg, $n(\%)$ & $5(1-7)$ \\
\hline
\end{tabular}

monocyte recruitment and subsequently the necrotic core macrophages in an atherogenic mouse model [42]. In agreement with these findings, in our study we found that PCSK9i was able to ameliorate the inflammatory state by reducing MHR in FH subjects.

PWV is a novel cardiovascular biomarker widely used in clinical practice and is a strong predictor of ASCVD in the general population [44]. It is known that statin therapy is able to reduce LDL-C as well as inflammatory profile [43, 44]; thus, these effects may better explain the statin promoted reduction of PWV in the general population [45]; thanks to these properties, statin is the first LDL-C lowering strategy in all subjects, in particular in FH subjects [46].
However, despite the use of statins, a high prevalence of premature ASCVD has been reported in FH subjects [47]. Therefore, the addition of novel lipid-lowering therapies such PCSK9-i may be helpful in reducing ASCVD risk in FH subjects. In this context, in our study we demonstrated that PCSK9-i reduced PWV and probably this effect may be the result of LDL-C and MHR reductions; in line with these considerations, in our study we found that $\Delta \mathrm{PWV}$ was significantly associated with $\Delta$ LDL-C, $\Delta \mathrm{NC}$ and $\Delta \mathrm{MHR}$.

We also found that PCSK9-i significantly reduced LDL-C by $49.61 \%$; this was in line with previous finding by Hollstein et al. who showed a similar LDL-C decrease in a real-world setting of subjects at high ASCVD risk [48]. Furthermore, in our study a $20.4 \%$ reduction of PWV was obtained by PCSK9-i therapy and this was in line with previous studies that showed a similar effect [49, 50]. In fact, Mandraffino et al. demonstrated that PCSK9-i significantly reduced PWV compared to ezetimibe in a real-word setting of FH subjects; furthermore, Cicero et al. found a significant improvement of PWV already after three months of PCSK9-i addition.

There are several limitations to our study; first, this was an open-label, prospective, observational but not randomized study and the PCSK9-i therapeutic strategy depended on a physician's decision. Moreover, the PCSK9-LOX1 axis was not studied. Furthermore, other parameters that may explain the interaction of LDL-C, inflammatory status and PWV such as flow mediated dilation and oxidative stress determination were not evaluated. The study population size was relatively small; however, we demonstrated a significant improvement of lipid and inflammatory profiles as well as PWV values after PCSK9-i therapy in FH subjects. These preliminary findings should be confirmed in a larger study population through defined diagnostic tools and statistical analyses. In conclusion, PCSK9-i therapy significantly ameliorated the lipid profile, inflammatory status and PWV values in a cohort of FH subjects; moreover, $\Delta \mathrm{PWV}$ was significantly associated with $\Delta$ LDL-C, $\Delta$ NC and $\Delta$ MHR. Our findings indicate the favorable role of this novel lipidlowering strategy on lipid and inflammatory profiles and PWV; however, a randomized controlled trial is required to evaluate the effect of PCSK9-i therapy on these pathological aspects in FH subjects. 
Table 2 Glucose, lipid, risk factor, liver and muscle enzyme profiles of the study population at baseline and after six months of add-on PCSK9-i therapy

\begin{tabular}{|c|c|c|c|c|}
\hline & FH subjects $(n=56)$ & FH subjects $(\mathrm{n}=56)$ & $\Delta$ & $\begin{array}{l}p \text { Value } \\
\text { between the } \\
\text { two groups }\end{array}$ \\
\hline & Baseline & 6-month add-on PCSK9-i & & \\
\hline \multicolumn{5}{|l|}{ Glucose profile } \\
\hline FPG, mg/dL & $93.86 \pm 9,31$ & $92.59 \pm 9,26$ & $-1.35 \%$ & 0.67 \\
\hline $\mathrm{HbA} 1 \mathrm{c}, \%$ & $5.62 \pm 0.47$ & $5.74 \pm 0.4$ & $2.14 \%$ & 0.24 \\
\hline Type 2 Diabetes, n (\%) & 3 & 3 & - & - \\
\hline \multicolumn{5}{|l|}{ Lipid profile } \\
\hline $\mathrm{TC}, \mathrm{mg} / \mathrm{dL}$ & $221.81 \pm 19.48$ & $143.39 \pm 18.35$ & $-35.35 \%$ & $<0.001$ \\
\hline $\mathrm{HDL}, \mathrm{mg} / \mathrm{dL}$ & $52.3 \pm 9.65$ & $54.45 \pm 9.22$ & $4.11 \%$ & 0.45 \\
\hline $\mathrm{TG}, \mathrm{mg} / \mathrm{dL}$ & $98.5(63.5-120.25)$ & $90.5(61.25-111.5)$ & $-8.12 \%$ & 0.27 \\
\hline LDL-C, mg/dL & $148.47 \pm 16.88$ & $74.81 \pm 16.33$ & $-49.61 \%$ & $<0.001$ \\
\hline LDL-C target, n (\%) & - & $24(42.9)$ & - & - \\
\hline Non-HDL-C, mg/dL & $169.12 \pm 16.66$ & $94.43 \pm 17.37$ & $-44.16 \%$ & $<0.001$ \\
\hline $\mathrm{ApoB}, \mathrm{mg} / \mathrm{dL}$ & $109.36 \pm 14.95$ & $61.94 \pm 13.99$ & $-43.36 \%$ & $<0.001$ \\
\hline ApoAI, m g/dL & $127.43 \pm 13.28$ & $129.76 \pm 12.86$ & $1.83 \%$ & 0.56 \\
\hline ApoB to ApoAI ratio & $0.87 \pm 0.25$ & $0.48 \pm 0.19$ & $-44.82 \%$ & $<0.001$ \\
\hline $\mathrm{Lp}(\mathrm{a}), \mathrm{nmol} / \mathrm{L}$ & $47.5(21.1-66.8)$ & $35.2(11.5-48.7)$ & $-25.89 \%$ & 0.34 \\
\hline \multicolumn{5}{|l|}{ Risk factors } \\
\hline Body mass index, $\mathrm{kg} / \mathrm{m}^{2}$ & $26.15 \pm 2.13$ & $26.1 \pm 2.11$ & $-0.19 \%$ & 0.87 \\
\hline Systolic BP, mmHg & $120.25 \pm 9.44$ & $118.15 \pm 9.91$ & $-1.75 \%$ & 0.45 \\
\hline Diastolic BP, mmHg & $71.5 \pm 10.1$ & $70.4 \pm 10.2$ & $-1.54 \%$ & 0.65 \\
\hline \multicolumn{5}{|l|}{ Liver and muscle enzymes } \\
\hline AST, U/L & $26.07 \pm 8.48$ & $25.03 \pm 8.21$ & $-3.99 \%$ & 0.76 \\
\hline ALT, U/L & $29.76 \pm 9.79$ & $28.53 \pm 9.51$ & $-4.15 \%$ & 0.71 \\
\hline CPK, U/L & $113.5(82.0-153.5)$ & $121.5(88-157.25)$ & $7.05 \%$ & 0.61 \\
\hline
\end{tabular}

Data are presented as mean $\pm \mathrm{SD}$, percentages, or median (interquartile range). PCSK9-I proprotein convertase subtilisin/kexin type 9 inhibitors, $F H$ familial hypercholesterolemia, FPG = fasting plasma glucose, $\mathrm{HbA1c}=$ glycated hemoglobin, $T C$ total cholesterol, $H D L$ high-density lipoprotein, $T G$ triglycerides, $L D L$ $C$ low-density lipoprotein cholesterol, $T G / H D L$ triglyceride to high-density lipoprotein ratio, $A p o B$ apolipoprotein B, $A p o A I$ apolipoprotein AI, $L p($ a) lipoprotein (a), $B P$ blood pressure, $A S T$ aspartate transaminase, $A L T$ alanine transaminase, $C P K$ creatine phosphokinase

\begin{tabular}{lllll}
\hline \multicolumn{2}{c}{ FH subjects $(n=56)$} & FH subjects $(n=56)$ & $\Delta$ & $\begin{array}{l}p \text { Value } \\
\text { between the } \\
\text { two groups }\end{array}$ \\
\multicolumn{1}{c}{ Baseline } & 6-month add-on PCSK9-i & \\
\hline White blood cell count & & & \\
$\mathrm{WBCC}, 10^{3} / \mu \mathrm{L}$ & $8.14 \pm 1.16$ & $7.18 \pm 1.26$ & $-11.79 \%$ & 0.07 \\
$\mathrm{NC}, 10^{3} / \mu \mathrm{L}$ & $5.21 \pm 1.04$ & $4.49 \pm 0.95$ & $-13.82 \%$ & $<0.05$ \\
$\mathrm{LC}, 10^{3} / \mu \mathrm{L}$ & $2.14 \pm 0.47$ & $2.11 \pm 0.76$ & $-0.01 \%$ & 0.67 \\
$\mathrm{MC}, 10^{3} / \mu \mathrm{L}$ & $0.62 \pm 0.17$ & $0.55 \pm 0.16$ & $-11.29 \%$ & 0.08 \\
Inflammatory profile & & & \\
$\mathrm{hs}-\mathrm{CRP}, \mathrm{mg} / \mathrm{dL}$ & $0.19(0.08-0.35)$ & $0.20(0.09-0.37)$ & $5.26 \%$ & 0.87 \\
$\mathrm{NLR}$ & $2.6 \pm 0.72$ & $2.5 \pm 0.7$ & $-3.85 \%$ & 0.45 \\
MHR & $11.56 \pm 4.14$ & $10.35 \pm 4.16$ & $-10.47 \%$ & $<0.05$ \\
\hline
\end{tabular}

Data are presented as mean $\pm \mathrm{SD}$, percentages, or median (interquartile range). PCSK9-i $=$ proprotein convertase subtilisin/kexin type 9 inhibitors, $F H$ familial hypercholesterolemia, $W B C C$ white blood cell count, $N C$ neutrophil count, $L C$ lymphocyte count, $M C$ monocyte count, $h s$ - $C R P$ high-sensitivity C-reactive protein, NLR = neutrophil-to-lymphocyte ratio, $M H R=$ monocyte-to-high-density lipoprotein ratio 
Fig. 2 PWV values of the study population after six months of add-on PCSK9-i. PWV = pulse wave velocity, PCSK9-i $=$ proprotein convertase subtilisin/ kexin type 9 inhibitors. $* \mathrm{P}$ value $<0.01$ vs baseline

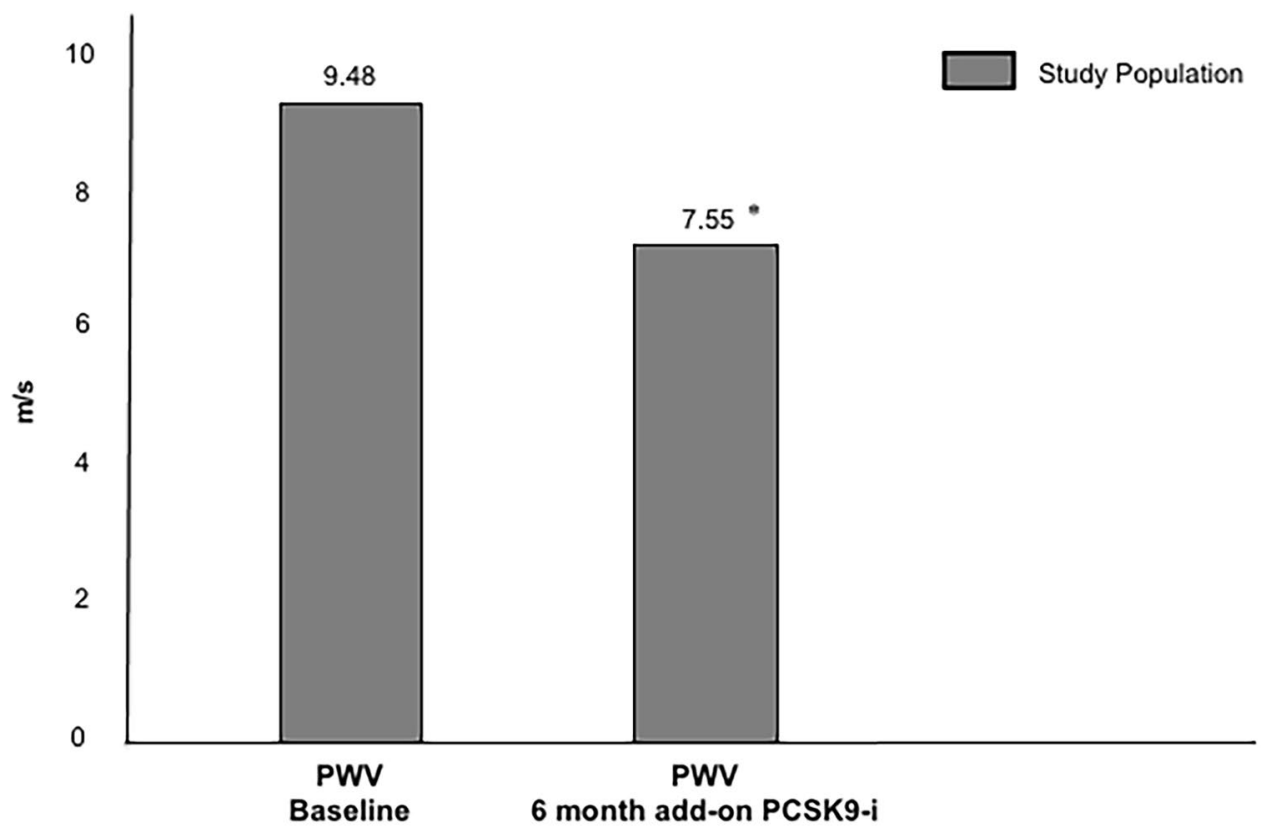

Table 4 Simple linear regression analyses evaluating $\triangle \mathrm{PWV}$ as a dependent variable

\begin{tabular}{lll}
\hline Dependent variable & $\Delta$ PWV & \\
\cline { 2 - 3 } Independent variable & Coefficient $\beta$ & $P$ value \\
\hline$\Delta$ LDL-C, $\%$ & $1.635 \pm 0.165$ & $<0.01$ \\
$\Delta$ NC, $\%$ & $1.249 \pm 0.110$ & $<0.05$ \\
$\Delta$ MHR, $\%$ & $1.283 \pm 0.112$ & $<0.05$ \\
\hline
\end{tabular}

$\triangle P W V$ change of pulse wave velocity from baseline for all the PCSK9-i therapy duration, $\triangle L D L-C$ change of low-density lipoprotein cholesterol from baseline for all the PCSK9-i therapy duration, $\triangle N C$ change of neutrophil count from baseline for all the PCSK9-i therapy duration, $\triangle M H R$ change of monocyte-to-high-density lipoprotein ratio from baseline for all the PCSK9-i therapy duration

Acknowledgements FP is the guarantor of this work and, as such, had full access to all the data in the study and takes responsibility for the integrity of the data and the accuracy of the data analysis. All authors approved the final version. The authors wish to thank the Department of Clinical and Experimental Medicine for financial support in the context of the 2016/2018 Department Research Plan of University of Catania (project \#A). Genetic analysis was carried out within the LIPIGEN study, an initiative of the SISA Foundation. The authors wish to thank the Scientific Bureau of the University of Catania for language support.

Author contributions R.S., F.P. and S.P. made substantial contributions to the conception and design, and they wrote and drafted the article; moreover, A.D.P., A.M.R., F.P. and S.P. critically revised the manuscript for intellectual content; R.S. performed PWV evaluation; A.D.P. and V.F. participated in the patient selection and recruitment, in the data collection and interpretation; R.S. performed the statistical analysis. R.S., A.D.P., F.P. and S.P. approved the manuscript to be submitted.

Funding Open access funding provided by Università degli Studi di Catania within the CRUI-CARE Agreement. This research did not receive any specific grant from funding agencies in the commercial or not-for-profit sectors.

\section{Compliance with ethical standards}

Conflict of interest The authors declare that they have no conflict of interest.

Ethical approval This study was approved by the local ethics committee in accordance with the ethical standards of the institutional and national research committees and with the 1964 Declaration of Helsinki and its later amendments or comparable ethical standards. This article does not contain any studies with animals performed by any of the authors.

Informed consent.

Informed consent was obtained from each participant enrolled in the study.

Open Access This article is licensed under a Creative Commons Attribution 4.0 International License, which permits use, sharing, adaptation, distribution and reproduction in any medium or format, as long as you give appropriate credit to the original author(s) and the source, provide a link to the Creative Commons licence, and indicate if changes were made. The images or other third party material in this article are included in the article's Creative Commons licence, unless indicated otherwise in a credit line to the material. If material is not included in the article's Creative Commons licence and your intended use is not permitted by statutory regulation or exceeds the permitted use, you will need to obtain permission directly from the copyright holder. To view a copy of this licence, visit http://creativecommons.org/licenses/by/4.0/. 


\section{References}

1. Falk E (2006) Pathogenesis of Atherosclerosis. J Am Coll Cardiol 47:C7-C12

2. Ference BA, Ginsberg HN, Graham I et al (2017) Low-density lipoproteins cause atherosclerotic cardiovascular disease. 1. Evidence from genetic, epidemiologic, and clinical studies. A consensus statement from the European Atherosclerosis Society Consensus Panel. Eur Heart J 38:2459-2472. https://doi.org/10. 1093/eurheartj/ehx144

3. Tardif J-C, Kouz S, Waters DD et al (2019) Efficacy and safety of low-dose colchicine after myocardial infarction. N Engl J Med 381:2497-2505. https://doi.org/10.1056/nejmoa1912388

4. Ridker PM, MacFadyen JG, Glynn RJ et al (2020) Comparison of interleukin-6, C-reactive protein, and low-density lipoprotein cholesterol as biomarkers of residual risk in contemporary practice: secondary analyses from the cardiovascular inflammation reduction trial. Eur Heart J 41:2952-2961. https://doi.org/10. 1093/eurheartj/ehaa160

5. Baragetti A, Catapano AL, Magni P (2020) Multifactorial activation of nlrp3 inflammasome: Relevance for a precision approach to atherosclerotic cardiovascular risk and disease. Int J Mol Sci 21:1-13

6. Bernardi S, Marcuzzi A, Piscianz E et al (2018) The complex interplay between lipids, immune system and interleukins in cardio-metabolic diseases. Int. J. Mol, Sci, p 19

7. Charakida M, Tousoulis D, Skoumas I et al (2009) Inflammatory and thrombotic processes are associated with vascular dysfunction in children with familial hypercholesterolemia. Atherosclerosis 204:532-537. https://doi.org/10.1016/j.atherosclerosis. 2008.09.025

8. Burden G, Roth GA, Mensah GA et al (2020) Global burden of cardiovascular diseases and risk factors, 1990-2019: update from the gbd 2019 study. J Am Coll Cardiol. https://doi.org/10. 1016/j.jacc.2020.11.010

9. Wang X, Zhang G, Jiang X et al (2014) Neutrophil to lymphocyte ratio in relation to risk of all-cause mortality and cardiovascular events among patients undergoing angiography or cardiac revascularization: A meta-analysis of observational studies. Atherosclerosis 234:206-213

10. Zhou Y, Wang L, Jia L et al (2020) The monocyte to high-density lipoprotein cholesterol ratio in the prediction for atherosclerosis: a retrospective study in adult chinese participants. Lipids. https:// doi.org/10.1002/lipd.12276

11. Averna M, Cefalù AB, Casula M et al (2017) Familial hypercholesterolemia: the Italian atherosclerosis society network (LIPIGEN). Atheroscler Suppl. https://doi.org/10.1016/j.atherosclerosis sup.2017.07.001

12. Pirillo A, Garlaschelli K, Arca M et al (2017) Spectrum of mutations in Italian patients with familial hypercholesterolemia: new results from the LIPIGEN study. Atheroscler Suppl. https://doi. org/10.1016/j.atherosclerosissup.2017.07.002

13. Scicali R, Di Pino A, Urbano F et al (2019) Analysis of S100A12 plasma levels in hyperlipidemic subjects with or without familial hypercholesterolemia. Acta Diabetol 56:899-906. https://doi.org/ 10.1007/s00592-019-01338-1

14. Casula M, Olmastroni E, Pirillo A et al (2018) Evaluation of the performance of Dutch Lipid Clinic Network score in an Italian FH population: The LIPIGEN study. Atherosclerosis 277:413-418. https://doi.org/10.1016/j.atherosclerosis.2018.08.013

15. Hu P, Dharmayat KI, Stevens CAT et al (2020) Prevalence of familial hypercholesterolemia among the general population and patients with atherosclerotic cardiovascular disease. Circulation
141:1742-1759. https://doi.org/10.1161/CIRCULATIONAHA. 119.044795

16. Benito-Vicente A, Uribe KB, Jebari S et al (2018) Familial hypercholesterolemia: the most frequent cholesterol metabolism disorder caused disease. Int J Mol Sci 19:3426

17. Dixon DL, Trankle C, Buckley L et al (2016) A review of PCSK9 inhibition and its effects beyond LDL receptors. J Clin Lipidol 10:1073-1080

18. Scicali R, Di Pino A, Ferrara V et al (2018) New treatment options for lipid-lowering therapy in subjects with type 2 diabetes. Acta Diabetol. https://doi.org/10.1007/s00592-017-1089-4

19. Schwartz GG, Steg PG, Szarek M et al (2018) Alirocumab and cardiovascular outcomes after acute coronary syndrome. N Engl J Med 379:2097-2107. https://doi.org/10.1056/NEJMoa1801174

20. Sabatine MS, Giugliano RP, Keech AC et al (2017) Evolocumab and clinical outcomes in patients with cardiovascular disease. $\mathrm{N}$ Engl J Med 376:1713-1722. https://doi.org/10.1056/NEJMoa1615 664

21. Galema-Boers AMH, Lenzen MJ, Sijbrands EJ, Roeters van Lennep JE (2017) Proprotein convertase subtilisin/kexin 9 inhibition in patients with familial hypercholesterolemia: Initial clinical experience. J Clin Lipidol 11:674-681. https://doi.org/10.1016/j. jacl.2017.02.014

22. Scicali R, Di Pino A, Platania R et al (2018) Detecting familial hypercholesterolemia by serum lipid profile screening in a hospital setting: clinical, genetic and atherosclerotic burden profile. Nutr Metab Cardiovasc Dis. https://doi.org/10.1016/j.numecd.2017.07. 003

23. Scicali R, Rosenbaum D, Di Pino A et al (2018) An increased waist-to-hip ratio is a key determinant of atherosclerotic burden in overweight subjects. Acta Diabetol 55:741-749. https://doi.org/ 10.1007/s00592-018-1144-9

24. Scicali R, Di Pino A, Urbano F et al (2020) Analysis of steatosis biomarkers and inflammatory profile after adding on PCSK9 inhibitor treatment in familial hypercholesterolemia subjects with nonalcoholic fatty liver disease: a single lipid center real-world experience. Nutr Metab Cardiovasc Dis. https://doi.org/10.1016/j. numecd.2020.11.009

25. Urbano F, Di Pino A, Scicali R et al (2019) Impaired glucagon suppression and reduced insulin sensitivity in subjects with prediabetes undergoing atorvastatin therapy. Eur J Endocrinol 181:579-590. https://doi.org/10.1530/EJE-19-0173

26. Scicali R, Giral P, Gallo A et al (2016) HbA1c increase is associated with higher coronary and peripheral atherosclerotic burden in non diabetic patients. Atherosclerosis. https://doi.org/10.1016/j. atherosclerosis.2016.11.003

27. Catapano AL, Graham I, De Backer G et al (2016) 2016 ESC/ EAS Guidelines for the management of dyslipidaemias. Eur Heart J 37:2999-3058. https://doi.org/10.1093/eurheartj/ehw272

28. Scicali R, Russo GI, Di Mauro M et al (2020) Analysis of arterial stiffness and sexual function after adding on pcsk9 inhibitor treatment in male patients with familial hypercholesterolemia: a single lipid center real-world experience. J Clin Med 9:3597. https://doi. org/10.3390/jcm9113597

29. Mandraffino G, Scicali R, Squadrito G (2020) Author's reply to: Arterial stiffness improvement after adding on PCSK9 inhibitors in patients with familial hypercholesterolemia, a letter from Papaioannou and colleagues. J Clin Lipidol 14:543

30. Spadaro L, Noto D, Privitera G et al (2014) Apolipoprotein AI and HDL are reduced in stable cirrhotic patients with adrenal insufficiency: a possible role in glucocorticoid deficiency. Scand J Gastroenterol. https://doi.org/10.3109/00365521.2014.985707

31. Scicali R, Di Pino A, Pavanello C et al (2019) Analysis of HDLmicroRNA panel in heterozygous familial hypercholesterolemia 
subjects with LDL receptor null or defective mutation. Sci Rep. https://doi.org/10.1038/s41598-019-56857-2

32. Scicali R, Giral P, D'Erasmo L et al (2020) High TG to HDL ratio plays a significant role on atherosclerosis extension in prediabetes and newly diagnosed type 2 diabetes subjects. Diabetes Metab Res Rev. https://doi.org/10.1002/dmrr.3367

33. Di Pino A, Urbano F, Scicali R et al (2019) 1 h Postload Glycemia Is associated with low endogenous secretory receptor for advanced glycation end product levels and early markers of cardiovascular disease. Cells 8:910. https://doi.org/10.3390/cells8080910

34. Flores-Gomez D, Bekkering S, Netea MG, Riksen NP (2020) Trained Immunity in atherosclerotic cardiovascular disease. Arterioscler Thromb Vasc Biol. https://doi.org/10.1161/atvbaha.120. 314216

35. Mitroulis I, Hajishengallis G, Chavakis T (2020) Trained immunity and cardiometabolic disease. Arterioscler Thromb Vasc Biol. https://doi.org/10.1161/atvbaha.120.314215

36. Rahman T, Hamzan NS, Mokhsin A et al (2017) Enhanced status of inflammation and endothelial activation in subjects with familial hypercholesterolaemia and their related unaffected family members: a case control study. Lipids Health Dis. https://doi.org/ 10.1186/s12944-017-0470-1

37. Shapiro MD, Fazio S (2017) PCSK9 and atherosclerosis - lipids and beyond. J Atheroscler Thromb 24:462-472

38. Ding Z, Liu S, Wang X et al (2015) Cross-Talk between LOX-1 and PCSK9 in vascular tissues. Cardiovasc Res 107:556-567. https://doi.org/10.1093/cvr/cvv178

39. Li S, Guo YL, Xu RX et al (2014) Association of plasma PCSK9 levels with white blood cell count and its subsets in patients with stable coronary artery disease. Atherosclerosis 234:441-445. https://doi.org/10.1016/j.atherosclerosis.2014.04.001

40. Ricci C, Ruscica M, Camera M et al (2018) PCSK9 induces a pro-inflammatory response in macrophages. Sci Rep. https://doi. org/10.1038/s41598-018-20425-x

41. Sahebkar A, Di Giosia P, Stamerra CA et al (2016) Effect of monoclonal antibodies to PCSK9 on high-sensitivity C-reactive protein levels: A meta-analysis of 16 randomized controlled treatment arms. Br J Clin Pharmacol 81:1175-1190. https://doi.org/ 10.1111/bcp. 12905

42. Kühnast S, Van Der Hoorn JWA, Pieterman EJ et al (2014) Alirocumab inhibits atherosclerosis, improves the plaque morphology, and enhances the effects of a statin. J Lipid Res 55:21032112. https://doi.org/10.1194/jlr.M051326
43. Baigent C, Blackwell L, Emberson J et al (2010) Efficacy and safety of more intensive lowering of LDL cholesterol: a metaanalysis of data from 170000 participants in 26 randomised trials. Lancet 376:1670-1681. https://doi.org/10.1016/S0140-6736(10) 61350-5

44. Albert MA, Danielson E, Rifai N, Ridker PM (2001) Effect of statin therapy on C-reactive protein levels: the pravastatin inflammation/CRP evaluation (PRINCE): a randomized trial and cohort study. J Am Med Assoc 286:64-70. https://doi.org/10.1001/jama. 286.1.64

45. D'elia L, La Fata E, Iannuzzi A, Rubba PO, (2018) Effect of statin therapy on pulse wave velocity: a meta-analysis of randomized controlled trials. Clin Exp Hypertens 40:601-608. https://doi.org/ 10.1080/10641963.2017.1411498

46. Casula M, Gazzotti M, Bonaiti F et al (2020) Reported muscle symptoms during statin treatment among Italian dyslipidemic patients in the real-life setting: the prosisa study. J Intern Med. https://doi.org/10.1111/joim.13219

47. Gulizia MM, Maggioni AP, Abrignani MG et al (2020) Prevalence of familial hypercholeSTerolaemia $(\mathrm{FH})$ in Italian patients with coronary artERy disease: The poster study. Atherosclerosis 308:32-38. https://doi.org/10.1016/j.atherosclerosis.2020.07.007

48. Hollstein T, Kassner U, Grenkowitz T et al (2020) PCSK9 inhibitors in a German single-center clinical practice: real-world treatment of patients at high cardiovascular risk Over 68 weeks. Am J Cardiovasc Drugs. https://doi.org/10.1007/s40256-020-00411-3

49. Mandraffino G, Scicali R, Rodríguez-Carrio J et al (2020) Arterial stiffness improvement after adding on PCSK9 inhibitors or ezetimibe to high-intensity statins in patients with familial hypercholesterolemia: a two-lipid center real-world experience. J Clin Lipidol. https://doi.org/10.1016/j.jacl.2020.01.015

50. Cicero AFG, Toth PP, Fogacci F et al (2019) Improvement in arterial stiffness after short-term treatment with PCSK9 inhibitors. Nutr. Metab. Cardiovasc, Dis

Publisher's Note Springer Nature remains neutral with regard to jurisdictional claims in published maps and institutional affiliations. 